\title{
Hypomethylation of paternal DNA in the late mouse zygote is not essential for development
}

\author{
ZBIGNIEW POLANSKII,2,*, NAMI MOTOSUGI ${ }^{1}$, CHIZUKO TSURUMI ${ }^{1, \#, ~ T A K A S H I ~ H I I R A G I ~}{ }^{1}$ and STEFFEN \\ HOFFMANN 1 ,\#\# \\ ${ }^{1}$ Department of Developmental Biology, Max Planck Institute of Immunobiology, Freiburg, Germany and \\ ${ }^{2}$ Department of Genetics and Evolution, Institute of Zoology, Jagiellonian University, Krakow, Poland
}

\begin{abstract}
Global demethylation of DNA which marks the onset of development occurs asynchronously in the mouse; paternal DNA is demethylated at the the zygote stage, whereas maternal DNA is demethylated later in development. The biological function of such asymmetry and its underlying mechanisms are currently unknown. To test the hypothesis that the early demethylation of male DNA may be associated with protamine-histone exchange, we ,used round spermatids, whose DNA is still associated with histones, for artificial fertilization (round spermatid injection or ROSI), and compared the level of methylation of metaphase chromosomes in the resulting zygotes with the level of methylation in zygotes obtained after fertilization using mature sperm heads (intracytoplasmic sperm injection or ICSI). In contrast to ICSI-derived zygotes, ROSI-derived zygotes possessed only slightly demethylated paternal DNA. Both types of zygotes developed to term with similar rates which shows that hypomethylation of paternal DNA at the zygotic metaphase is not essential for full development in mice. Incorporation of exogenously expressed histone H2BYFP into paternal pronuclei was significantly higher in ICSI-derived zygotes than in ROSI-derived zygotes. Surprisingly, in the latter the incorporation of histone H2BYFP into the paternal pronucleus was still significantly higher than into the maternal pronucleus, suggesting that some exchange of chromatin-associated proteins occurs not only after ICSI but also after ROSI. This may explain why after ROSI, some transient demethylation of paternal DNA occurs early after fertilization, thus providing support for the hypothesis regarding the link between paternal DNA demethylation and protamine/histone exchange.
\end{abstract}

KEY WORDS: mouse, zygote, DNA demethylation, paternal DNA, protamine/histone exchange

\section{Introduction}

In the mammalian germ cell line, global DNA demethylation, which occurs at the time of primordial germ cell migration into the genital ridges is followed by methylation during later stages of gametogenesis (Morgan et. al., 2004). Thus, the DNA of both parental gametes is highly methylated at the time of fertilization but undergoes profound demethylation at the onset of development. Surprisingly in mouse and some other mammalian species this process occurs asynchronously; the paternal DNA being demethylated at the zygote stage but the maternal one later in the development (Mayer et al., 2000a; Dean et al., 2001; Xu et al.,
2005). It was proposed that such asymmetry might be essential for the development (Mayer et al., 2000a; Barton et al., 2001; Shi and Haaf, 2002: Xu et al., 2005). Until present, however, neither the biological function of such asymmetry nor its underlying mechanism was clarified.

During final stages of spermatogenesis after passing the round spermatid stage the histones in the nuclei of elongating spermatids are replaced by protamines ensuring the tight packing of DNA in the sperm head (Sassone-Corsi, 2002). Shortly after fertiliza-

Abbreviations used in this paper: ICSI, intracytoplasmic sperm injection; ROSI, round spermatid injection, BrdU, bromodeoxyuridine.

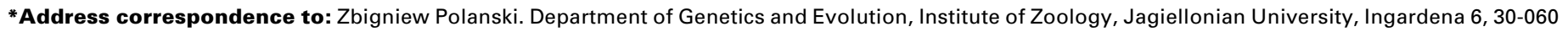
Krakow, Poland. Fax: +48-12-634-3716. e-mail: pola@iz.uj.edu.pl Web: http://www.binoz.uj.edu.pl/iz/index.php?idz=zakl_prac\&id=23
}

\#Present address: Hematology and Oncology Department, University of Freiburg Medical Center, Hugstetter Str. 55, 79106 Freiburg, Germany

\#\#Present address: ProQinase GmbH, Breisacher Str. 117, 79106 Freiburg, Germany

Published online: 14 February 2008

0214-6282/2008/\$35.00

(C) UBC Press

Printed in Spain 
tion, at the time coincident with demethylation of male DNA, the protamines bound to sperm DNA are again replaced by the histones (Santos et al., 2002). It seems that in the course of such a protein exchange the conformation of paternal DNA loosens extensively (Rodman et al., 1981). Supposedly, this may result in easier accessibility of the putative, yet unidentified, DNA demethylase to the paternal but not maternal DNA. If this is the case then after fertilization the histone-associated DNA of round spermatids should be resistant to global demethylation characteristic for protamin-associated DNA of the mature sperm. In this study we have compared the level of methylation of paternal DNA in mouse zygotes obtained after injection of round spermatids (ROSI) or injection of sperm heads (ICSI).

\section{Results and Discussion}

Male mice treated with BrdU were used as donors of sperm or round spermatids. One-cell embryos produced by ICSI or ROSI were cultured to metaphase stage followed by preparation of metaphase chromosomes. Chromosomes were also prepared from one-cell metaphase embryos from two control groups. The first group consisted of diploid parthenogenotes obtained by activation of MIl oocytes in the presence of cytochalasin B. The second control group comprised diploid gynogenetic embryos produced by removing male pronucleus from in vivo fertilized zygote and replacing it with the female pronucleus transferred from another zygote. Embryos were labeled using well characterized antibody recognizing methylated cytosine. In case of ROSI and ICSI embryos additional labeling with anti BrdU antibody allowed to discriminate between paternal and maternal set of chromosomes. In pilot studies the chromosome spreads were labeled with the specific probe against whole $Y$ chromosome to identify male chromosome complement. Since such procedure allows distinction between parental genomes only in $50 \%$ of zygotes (these with XY combination of sex chromosome pair), it was replaced with BrdU labeling.

As expected, in control embryos both haploid sets of chromosomes exhibited similar level of methylation consistent with the fact that maternal DNA does not undergo demethylation in onecell embryos (Fig. 1A, B). This confirmed the validity of the procedure used to assess the methylation level. As shown in Fig. $1 \mathrm{~B}$ the variance in the methylation of the two maternal chromosome sets was higher in digynic embryos (where the chromo-

A

D
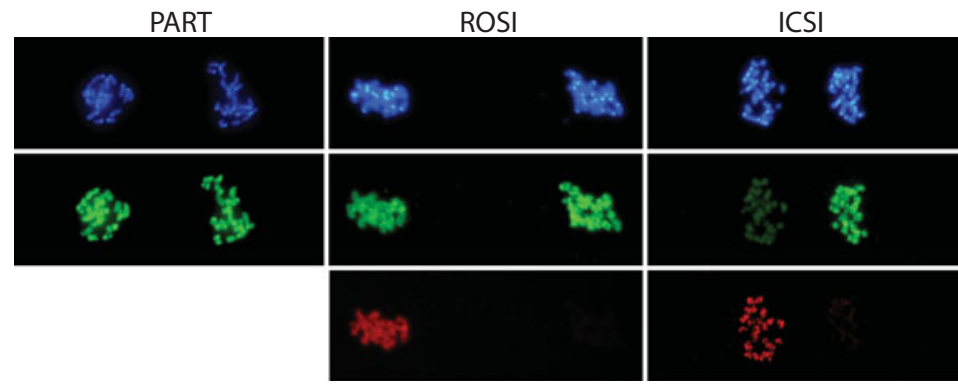

DAPI

5-methyl cytosine

BrdU
B

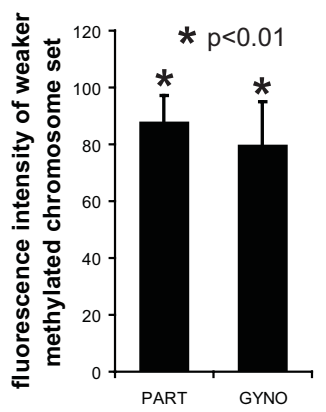

C

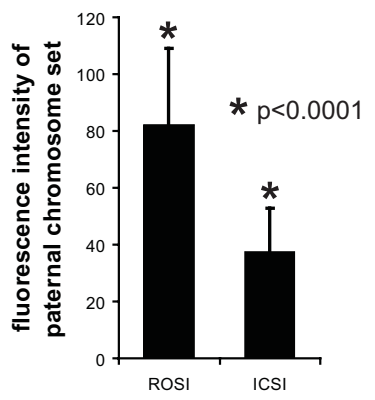

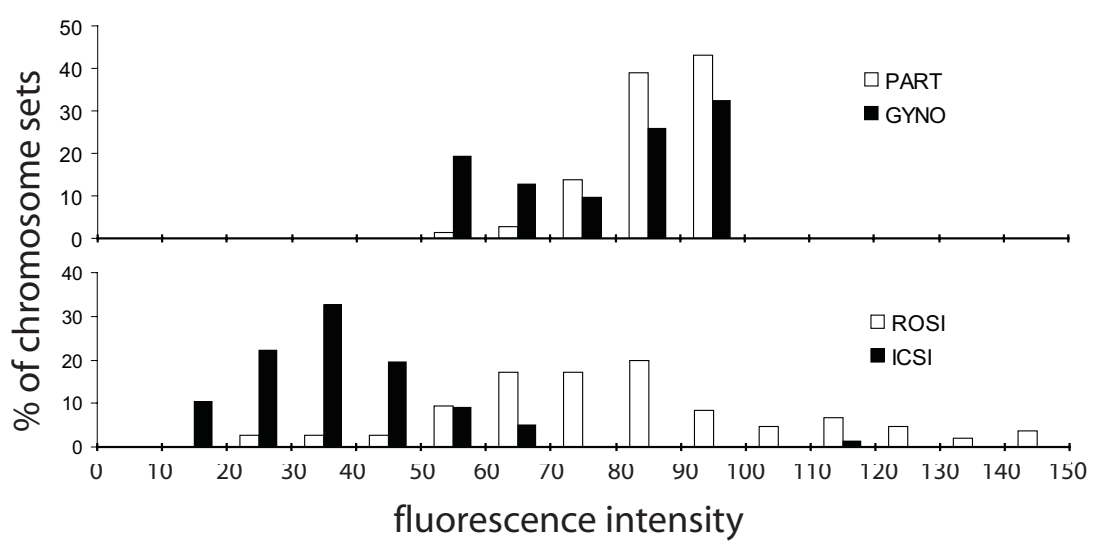

Fig. 1. DNA methylation in different types of metaphase stage one-cell embryos. (A) Typical sets of chromosomes. Labeling with BrdU identifies the paternal set of chromosomes. (B) Difference in methylation level between two maternal chromosome sets in parthenogenetic (PART, N = 71) and digynic embryos (GYNO, N = 31). Bars represent the relative level of methylation of the lesser methylated chromosome set compared to $100 \%$ methylation in the higher methylated set. In the digynic embryos, the average DNA methylation of the weaker methylated set is significantly lower than in parthenogenetic embryos (t-test). (C) The relative methylation (\% of methylation scored for maternal chromosome set) of paternal DNA in ROSI (N = 106) and ICSI ( $N=77)$ zygotes differs significantly (t-test). (D) Distribution of one-cell embryos revealing different level of methylation of paternal chromosome set (ROSI and ICSI) or weaker methylated chromosome set (gyno and part). (B,C) Mean $\pm S D$ is shown.

some sets originate from two different oocytes) than in parthenogenotes (both chromosome sets originate from one set of chromosomes of metaphase II oocyte). This higher heterogeneity observed in digynic embryos indicates that the level of DNA methylation differs to some extent between individual oocytes.

Analysis of ROSI and ICSI zygotes revealed the striking difference in the level of DNA methylation of male metaphase chromosomes. On average, DNA methylation of paternal chro- 


\section{TABLE 1}

\section{DEVELOPMENT IN VIVO AFTER TRANSFER OF 2-CELL EMBRYOS FOLLOWED ICSI OR ROSI}

\begin{tabular}{ccccc} 
Treatment & $\begin{array}{c}\text { No. of transferred } \\
\text { embryos/recipients }\end{array}$ & $\begin{array}{c}\text { No. of pregnant } \\
\text { recipients }\end{array}$ & $\begin{array}{c}\text { No. (\%) } \\
\text { of live born }\end{array}$ & $\begin{array}{c}\text { \% of live born from } \\
\text { pregnant recipients }\end{array}$ \\
\hline ICSI & $36 / 4$ & $3(26)$ & $9(25)$ & 35 \\
ROSI & $39 / 5$ & $3(28)$ & $12(31)$ & 43 \\
\hline
\end{tabular}

*the number of embryos transferred into foster mothers which became pregnant is shown in parentheses. Difference in the number of embryos developed to term was not significant $(P>0.5)$ for all foster mothers as well as foster mothers which became pregnant.

mosomes in ROSI and ICSI zygotes amounted respectively to the $82 \%$ and $37 \%$ of methylation observed in the female chromosomes (Fig. 1A, C), thus confirming predicted weak demethylation in the round spermatid chromatin. Comparison of the distribution of the zygotes with different level of methylation of paternal chromosomes shows only slight overlap between ROSI and ICSI (Fig. 1D). For example, in as high as $82 \%$ of ROSI zygotes but only in $7 \%$ of ICSI zygotes the paternal chromosomes revealed DNA methylation higher than $60 \%$ of methylation observed in maternal chromosomes (Fig. 1D). Moreover, the distribution of paternal DNA methylation in ROSI embryos fits well with the distribution of DNA methylation in digynic embryos since in $81 \%$ of the latter embryos the DNA methylation of less methylated chromosome set exceeded $60 \%$ of methylation of the other chromosome set (Fig. 1D). These data clearly show that the methylation level of the paternal DNA of metaphase chromosomes of one-cell ROSI embryos, but not one-cell ISCI ones, lies in the range of methylation observed for DNA of maternal metaphase chromosomes.

Injection of mRNA coding for histone H2B-YFP followed by ROSI or ICSI enabled us to assess the dynamics of histone incorporation into the two types of male pronucleus. The intensity of YFP signal detected in male pronucleus was significantly higher in ICSI zygotes than in ROSI zygotes (Fig. 2A, B) consistent with an extensive exchange of protamines to histones occuring in sperm chromatin shortly after fertilization. Surprisingly, the male pronucleus incorporated significantly more histone H2BYFP than female pronucleus not only in ICSI but also in ROSI zygotes (Fig. 2B).

Finally, in a portion of experiments, the ICSI and ROSI embryos instead of being subjected to chromosome analysis were used for embryo transfer. As presented in Table 1 the similar proportion of transferred ROSI and ICSI embryos gave

A

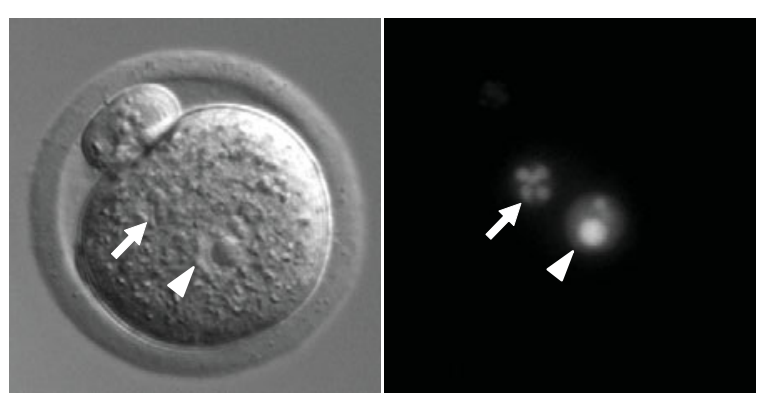

Fig. 2. Incorporation of exogenous histone H2BYFP into pronuclei. (A) Typical ICSI zygote expressing injected construct. Arrow and arrowhead indicate female and male pronucleus, respectively. (B) The average level of histone incorporation. The sig- live-born pups.

Our results show that in the majority of zygotes obtained from ROSI the level of DNA methylation of metaphase chromosomes is high and comparable with the level of methylation of maternal chromosomes. This confirms the recent report (Kishigami et al., 2006), which is in striking contrast with the observations that in normal fertilization the sperm-originating DNA undergoes stable demethylation shortly after fertilization (Mayer et al., 2000a; Dean et al., 2001). We have also observed the much higher incorporation of histone H2BYFP into male pronucleus in ICSI zygotes than in ROSI ones. This clearly shows that an extensive remodeling of the male chromatin associated with exchange of protamines to histones after incorporation of sperm head (Santos et al., 2002) does not occur after incorporation of spermatid nucleus. These results are consistent with the hypothesis that the demethylation of male DNA in fertilized mouse egg may result from protein exchange-associated loosening of the chromatin structure which would make it easy accessible to the DNA demethylase. In contrast, the maternal chromatin or paternal chromatin originating from round spermatid nuclei and undergoing no or only limited remodeling would remain unaccessible (or of limited access) to such demethylase, and thus methylation of its DNA would remain high. However, it was recently reported that in the ROSI zygotes the male chromatin undergoes moderate DNA demethylation followed by its remethylation before reaching metaphase stage (Kishigami, et al., 2006). Such intermediate demethylation of paternal DNA may suggest some limited remodeling of the chromatin of the round spermatid in the oocyte cytoplasm resulting in partially enhanced accessibility of putative DNA demethylase. In fact, our observation that pronucleus formed from round spermatid nucleus incorporates more histone H2BYFP than pronucleus formed from maternal chromatin supports such explanation. The reason why such chromatin undergoes subsequent remethylation (Kishigami et al., 2006) remains unclear.

One of the crucial conditions affecting efficiency of development after ROSI lies in the proper time interval between oocyte activation and injection of round spermatid nucleus. In the careful study Kishigami and colleagues (2004) found that the highest rate of development to term was obtained when the injection of spermatid nucleus occurred 60-80 minutes after activation stimulus. In such conditions the efficiency of full-term development did not differ significantly from the efficiency of development after ICSI (54-56\% for ROSI embryos and 63\% for ICSI embryos, difference not significant $(P>0.4)$ when calculated using $\chi^{2}$ test nificance of the difference between female pronuclei and male pronuclei (ROSI or ICSI)

B

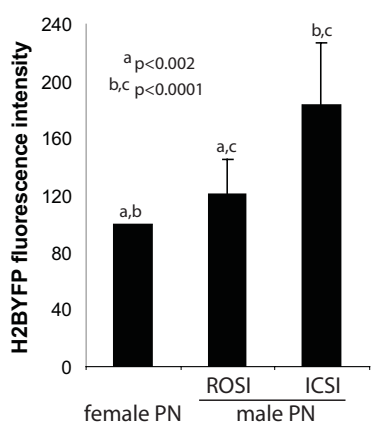

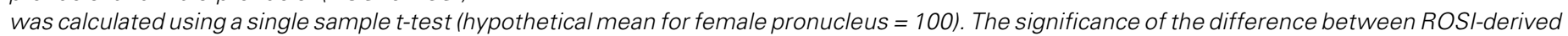
male pronuclei and ICSI-derived male pronuclei was calculated by t-test. 17 ROSI and 23 ICSI zygotes were analysed. Mean \pm SD is shown. 
applied for values obtained in that study). In our ROSI procedure we have adopted the 60-80 minutes time interval between activation and injection, which also resulted in the rate of development similar to ICSI embryos (Table 1). To our knowledge our report for the first time, combines, within the single study the analysis of chromosome methylation of ROSI zygotes with the efficiency of their development, thus directly addressing the issue of the importance of paternal DNA demethylation in the zygote. We should assume that, as demonstrated recently (Kishigami et al., 2006) the DNA of round spermatid chromatin underwent probably reasonable transient demethylation at the pronuclear stage. However, by the metaphase stage the methylation level was again high and comparable to the methylation level of maternal DNA. Nevertheless, such late stage zygotes with high global methylaton of paternal DNA developed to term with similar success rate as zygotes with low level of paternal DNA methylation obtained from ICSI. This clearly shows that hypomethylation of paternal DNA in the late zygotes is dispensable for development.

\section{Materials and Methods}

Female and male $\mathrm{F}_{1}$ hybrid mice $\mathrm{C} 57 \mathrm{BIXDBA} / 2$ were used throughout experiments. BrdU treatment was performed as described (Mayer et al., $2000 \mathrm{~b}$ ). The suspension of spermatogenic cells was obtained by squeezing the seminiferous tubules. Round spermatids were selected for ROSI according to the cell size and morphology (Kimura and Yanagimachi, 1995). Mature sperms were obtained from cauda epididymis.

Diploid parthenogenetic embryos were obtained by applying the activation protocol used for ROSI in the presence of cytochalasin B. Digynic gynogenetic embryos were produced by replacing the male pronuclei with the female pronuclei between in vivo fertilized zygotes, using previously described procedure for pronuclear transfer (McGrath and Solter, 1983; Polanski et al., 2005). ICSI was performed using piezoinjector (PrimeTech, Japan) according to Nagy et al. (2003). For ROSI we have followed the protocol described by Kishigami et al., applying the 60-80 minutes time interval between oocyte activation and spermatid injection. Histone H2BYFP was injected as described previously (Polanski et al., 2005) except that donor nucleus (sperm head or round spermatid nucleus) was not injected simultaneously with mRNA but 2 hours later. Images of embryos expressing histone H2BYFP were captured using AxioVision software (Zeiss) 5-8 hours after injection of sperm head or round spermatid.

Histone H2BYFP RNA was produced as described (Tsurumi et al., 2004).

Zygotes used for DNA methylation analysis or for assessing their developmental potential were checked 5-8 hours after injection of sperm cell nuclei and only those having second polar body and well developed two pronuclei were used for further experiments. After overnight culture the two-cell embryos were transferred into foster mothers as described (Nagy et al., 2003).

For DNA methylation analysis zygotes were cultured in medium supplemented with nocodazole and chromosome preparations were made next morning using Tarkowski's method (1965). Staining was performed according to Barton et al., (2001) using mouse primary antibody against 5-methyl-cytosine (Eurogentec, Belgium) and FITCconjugated goat antimouse secondary antibody (Santa Cruz Biotechnology). For identification of paternal chromosome set additional staining was performed applying rat antiBrdU primary antibody (Abcam) and TRITC-conjugated antirat secondary antibody (Sigma). Alternatively, staining with Cy3-conjugated whole $\mathrm{Y}$-chromosome specific probe (Cambio, UK) was performed according to instructions of manufacturer. AxioVision was used for acquisition of images.
Analysis of the fluorescence intensity was performed using Adobe Photoshop. In each embryo analyzed the virtual rectangle frame was created with the dimensions necessary to cover completely either of the single chromosome set. This rectangle was placed to cover the first chromosome set and the mean luminosity of the marked area was measured. Then, the rectangle was moved to cover the other chromosome set followed by measurement. The same procedure was used to estimate the intensity of fluorescence of histone H2BYFP in pronuclei, except that the measured area was circular.

\section{Acknowledgments \\ We thank Davor Solter for support and discussion.}

\section{References}

BARTON, S.C., ARNEY, K.L., SHI, W., NIVELEAU, A., FUNDELE, R., SURANI, M.A. and HAAF, T. (2001). Genome-wide methylation patterns in normal and uniparental early mouse embryos. Hum. Mol. Genet. 10: 2983-2987.

DEAN, W., SANTOS, F., STOJKOVIC, M., ZAKHARTCHENKO, V., WALTER, J., WOLF, E. and REIK, W. (2001). Conservation of methylation reprogramming in mammalian development: Aberrant reprogramming in cloned embryos. Proc. Natl. Acad. Sci. USA 98: 13734-13738.

KIMURA, Y. and YANAGIMACHI, R. (1995). Mouse oocytes injected with testicular spermatozoa or round spermatids can develop into normal offspring. Development 121: 2397-2405.

KISHIGAMI, S., VAN THUAN, N., HIKICHI, T., OHTA, H., WAKAYAMA, S. and WAKAYAMA, T. (2006). Epigenetic abnormalities of the mouse paternal zygotic genome associated with microinsemination of round spermatids. Dev. Biol. 289:195-205.

KISHIGAMI, S., WAKAYAMA, S., VAN THUAN, N. and WAKAYAMA, T. (2004). Similar Time Restriction for Intracytoplasmic Sperm Injection and Round Spermatid Injection into Activated Oocytes for Efficient Offspring Production. Biol. Reprod. 70: 1863-1869.

MAYER, W., NIVELEAU, A. WALTER, J., FUNDELE, R. and HAAF, T. (2000a). Demethylation of the zygotic paternal genome. Nature 403: 501-502.

MAYER, W., SMITH, A., FUNDELE, R. and HAAF, T. (2000b). Spatial separation of parental genomes in preimplantation mouse embryos. J. Cell Biol. 148: 629634.

MCGRATH, J. and SOLTER, D. (1983). Nuclear transplantation in the mouse embryo by microsurgery and cell fusion. Science 220: 1300-1302.

MORGAN, D.H., SANTOS, F. GREEN, K., DEAN, W. and REIK, W. (2005). Epigenetic reprogramming in mammals. Hum. Mol. Genet. Spec. No. 1: R47-58.

NAGY, A., GERTSENSTEIN, M., VINTERSTEN, K. and BEHRINGER, R. (Eds.). (2003). Manipulating the Mouse Embryo. A Laboratory Manual. Cold Spring Harbor Laboratory Press, Cold Spring Harbor, New York.

POLANSKI, Z. HOFFMANN, S. and TSURUMI, C. (2005). Oocyte nucleus controls progression through meiotic maturation. Dev. Biol. 281: 184-195.

RODMAN, T.C., PRUSLIN, F.H., HOFFMANN, H.P. and ALLFREY, V.G. (1981). Turnover of basic chromosomal proteins in fertilized eggs: a cytoimmunochemical study of events in vivo. J. Cell Biol. 90: 351-361.

SANTOS, F., HENDRICH, B., REIK, W. and DEAN, W. (2002). Dynamic reprogramming of DNA methylation in the early mouse embryo. Dev. Biol. 241: 172-182.

SASSONE-CORSI, P. (2002). Unique chromatin remodelling and transcriptional regulation in spermatogenesis. Science 296: 2176-2178.

SHI, W. and HAAF, T.(2002). Aberrant methylation patterns at the two-cell stage as an indicator of early developmental failure. Mol. Reprod. Dev. 63: 329-334.

TARKOWSKI, A.K. (1966). An air-drying method for chromosome preparations from mouse eggs. Cytogenetics 5, 394-400.

TSURUMI, C., HOFFMANN, S., GRAESER, R., GELEY, S. and POLANSKI, Z. (2004). The spindle assembly checkpoint is not essential for CSF arrest of mouse oocytes. J. Cell Biol. 167: 1037-1050.

XU, Y., ZHANG, J.J., GRIFO, J.A. and Krey, L.C. (2005). DNA methylation patterns in human tripronucleate zygotes. Mol. Hum. Reprod. 11: 167-171. 


\section{Related, previously published Int. J. Dev. Biol. articles}

See our recent Special Issue Developmental Biology in Poland edited by Kloc, Maleszewski and Tarkowski at: http://www.ijdb.ehu.es/web/contents.php?vol=52\&issue=2-3

See our Special Issue Mammalian Reproduction \& Development in honor of Anne McLaren and edited by Brigid Hogan at: http://www.ijdb.ehu.es/web/contents.php?vol=45\&issue $=3$

DNA methylation state is preserved in the sperm-derived pronucleus of the pig zygote

Young-Sun Jeong, Seungeun Yeo, Jung-Sun Park, Deog-Bon Koo, Won-Kyung Chang, Kyung-Kwang Lee and Yong-Kook Kang

Int. J. Dev. Biol. (2007) 51: 707-714

A hypothesis linking low folate intake to neural tube defects due to failure of post-translation methylations of the cytoskeleton

Natalie K. Björklund and Richard Gordon

Int. J. Dev. Biol. (2006) 50: 135-141

Histone methylation defines epigenetic asymmetry in the mouse zygote. Katharine L Arney, Siqin Bao, Andrew J Bannister, Tony Kouzarides and M Azim Surani

Int. J. Dev. Biol. (2002) 46: 317-320

Dynamic readjustment of parental methylation patterns of the 5 '-flank of the mouse $\mathrm{H} 19$ gene during in vitro organogenesis.

L Liang, C Kanduri, M Pilartz, K Svensson, J H Song, P Wentzel, U Eriksson and R Ohlsson

Int. J. Dev. Biol. (2000) 44: 785-790

Requirement of protamine for maintaining nuclear condensation of medaka (Oryzias latipes) spermatozoa shed into water but not for promoting nuclear condensation during spermatogenesis.

Y Shimizu, K Mita, M Tamura, K Onitake and M Yamashita

Int. J. Dev. Biol. (2000) 44: 195-199

Tissue-specific characterisation of DNA methylation in the gonadspecific proto-oncogene, c-mos, in the male laboratory mouse.

$\mathrm{C}$ Kanduri and R Raman

Int. J. Dev. Biol. (1999) 43: 91-94

Developmental-dependent DNA methylation of the IGF2 and H19 promoters is correlated to the promoter activities in human liver development.

X Li, S G Gray, F Flam, T Pietsch and T J Ekström

Int. J. Dev. Biol. (1998) 42: 687-693

DNA methylation and polyamines in embryonic development and cancer.

O Heby

Int. J. Dev. Biol. (1995) 39: 737-757

Imprinting.

D Solter

Int. J. Dev. Biol. (1998) 42: 951-954

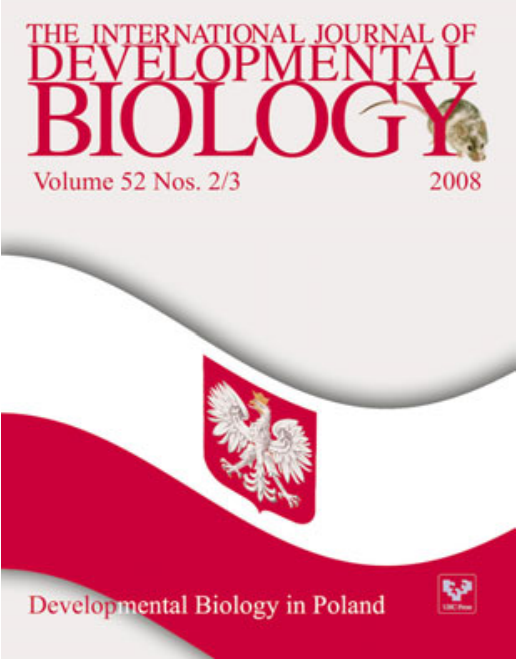

2006 ISI ${ }^{* \star}$ Impact Factor $=3.577^{\star *}$
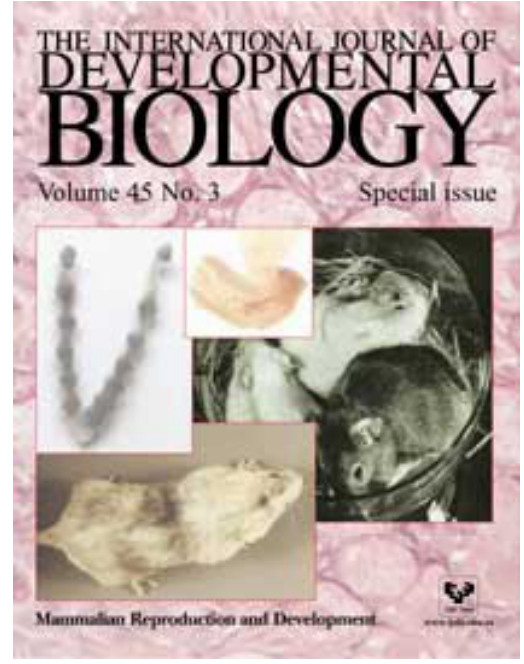